\title{
On-Ground and In-Orbit Characterisation Plan for the PLATO CCD Normal Cameras
}

\author{
J. P. D. Gow, ${ }^{\mathrm{a},{ }^{*}}$ D. Walton ${ }^{\mathrm{a}}$, A. Smith ${ }^{\mathrm{a}}$, M. Hailey ${ }^{\mathrm{a}}$, P. Curry ${ }^{\mathrm{a}}$ and T. Kennedy ${ }^{\mathrm{a}}$ \\ ${ }^{a}$ Mullard Space Science Laboratory, \\ University College London, Holmbury House, RH5 6NT, UK \\ E-mail: j.gow@ucl.ac.uk
}

\begin{abstract}
PLAnetary Transits and Ocillations (PLATO) is the third European Space Agency (ESA) medium class mission in ESA's cosmic vision programme due for launch in 2026. PLATO will carry out high precision un-interrupted photometric monitoring in the visible band of large samples of bright solar-type stars. The primary mission goal is to detect and characterise terrestrial exoplanets and their systems with emphasis on planets orbiting in the habitable zone, this will be achieved using light curves to detect planetary transits. PLATO uses a novel multi- instrument concept consisting of 26 small wide field cameras The 26 cameras are made up of a telescope optical unit, four Teledyne e2v CCD270s mounted on a focal plane array and connected to a set of Front End Electronics (FEE) which provide CCD control and readout. There are 2 fast cameras with high read-out cadence $(2.5 \mathrm{~s})$ for magnitude $\sim 4-8$ stars, being developed by the German Aerospace Centre and 24 normal $(\mathrm{N})$ cameras with a cadence of $25 \mathrm{~s}$ to monitor stars with a magnitude greater than 8 . The N-FEEs are being developed at University College London's Mullard Space Science Laboratory (MSSL) and will be characterised along with the associated CCDs. The CCDs and N-FEEs will undergo rigorous on-ground characterisation and the performance of the CCDs will continue to be monitored in-orbit. This paper discusses the initial development of the experimental arrangement, test procedures and current status of the N-FEE. The parameters explored will include gain, quantum efficiency, pixel response non-uniformity, dark current and Charge Transfer Inefficiency (CTI). The current in-orbit characterisation plan is also discussed which will enable the performance of the CCDs and their associated N-FEE to be monitored during the mission, this will include measurements of CTI giving an indication of the impact of radiation damage in the CCDs.
\end{abstract}

KEYWORDS: CCD; Characterisation; N-FEE; Normal Camera; PLATO.

\footnotetext{
* Corresponding author.
} 


\section{Contents}

1. Introduction $\quad 1$

1.1 Normal Front End Electronics and CCD Operation $\quad 2$

1.2 Radiation Damage Implications 3

2. On Ground Characterisation $\quad 5$

3. Inflight Test Strategy $\quad 6$

$\begin{array}{ll}3.1 \text { CTI Monitoring } & 7\end{array}$

3.2 Inflight Defect Identification $\quad 8$

4. Conclusions $\quad 9$

\section{Introduction}

PLAnetary Transits and Ocillations (PLATO) [1] is due for launch in 2026 and is the third medium class mission in the European Space Agencies (ESA) cosmic vision programme. The primary mission goal is to detect and characterise terrestrial exoplanets with emphasis on planets orbiting in the habitable zone. This will be achieved using high precision un-interrupted photometric monitoring (months to years) in the visible band of large samples of bright solartype stars. Asteroseismology analysis will also allow accurate stellar parameters and ages to be determined thus permitting an evolutionary study of planetary systems and the creation of a third dimension (age) to the Hertzsprung-Russell diagram. To achieve this PLATO will operate for a nominal 4 years, which could be extended to 6.5, at L2. PLATO will use a novel multiinstrument concept consisting of 26 small wide field cameras. Each camera is made up of a Telescope Optical Unit (TOU) [2], four Teledyne e2v CCD270s [3] mounted on a Focal Plane Array (FPA) produced by Instituto Nacional de Técnica Aeroespacial, and a set of Front End Electronics (FEE) to provide CCD control and readout. There are two fast (F) cameras for magnitude 4-8 stars, the F-FEE is being developed by the German Aerospace Centre, and twenty four normal $(\mathrm{N})$ cameras to monitor stars with a magnitude greater than 8 , the N-FEE is being developed at University College London's Mullard Space Science Laboratory (MSSL).

The un-interrupted monitoring of a large number of stars will enable PLATO to detect the reduction in a stars intensity caused by a transiting planet, described by the light curve, allowing the planet radii to be determined to around 3\% accuracy. Transit photometry was first demonstrated in planet HD 209458b [4], the planet was identified using radial velocity measurements and the light curve monitored prior to the predicted time of planetary transit. Although the probability of alignment for a transit is low this is countered by a wide field of view (2250 $\mathrm{deg}^{2}$ for PLATO) allowing a large number of stars to be monitored for transits, making this an efficient method to search for planets [5]. Stars with transits identified by PLATO will be targeted for ground based radial velocity measurements, which when combined with the orbital inclination from the light curve will enable the planets mass to an accuracy of 
around $10 \%$ to be determined. The use of the European Extremely Large Telescope will be included in the ground based part of the study and will enable planets identified by PLATO to be characterised further [6].

PLATO uses 104 CCD270s, a $4510 \times 4510$ device with $18 \mu \mathrm{m}$ square pixels with a typical Full Well Capacity (FWC) of $1050 \mathrm{ke}^{-}$, developed for PLATO by Teledyne e2v [3]. Two version of the CCD exist, a full frame device used in the normal cameras and a frame transfer device used in the fast cameras. The total image area (accounting for the optical shields in the fast camera CCDs) for PLATO will be $0.659 \mathrm{~m}^{2}$, over a factor 2 greater than ESAs Gaia spacecraft $\left(0.281 \mathrm{~m}^{2}\right)$ and over 4000 times larger than the first use of a CCD in space on board NASAs Galileo spacecraft [7].

While the CCDs and FEEs will be thoroughly tested to confirm conformance to requirments, the N-FEE/CCD and F-EEE/CCD combinations will undergo rigorous on ground characterisation. Moreover, it is necessary to continue to monitor the performance of the CCDs during the mission to maximise science return. This paper describes the CCD and its operation, the N-FEE being developed at MSSL and the initial development of the experimental arrangement and test procedures. The current in flight characterisation plan is also discussed, which will include monitoring the Charge Transfer Inefficiency (CTI) of the CCDs and the provision of trap parameters for charge transfer correction tools being developed by Laboratoire d'études spatiales et d'instrumentation en astrophysique (LESIA).

\subsection{Normal Front End Electronics and CCD Operation}

The function of the N-FEE, Figure 1, is to control and read-out the four CCD270s. All the CCDs are operated in exact synchronism to minimise the danger of coupled noise sources when CCDs are in close proximity. Both read-out ports of any one CCD are clocked and read-out at any one time with a pixel read-out rate of $3 \mathrm{MHz}$. Each of the four N-FEE CCDs are clocked and read-out in a rotating sequence at a cadence of $2.5 \mathrm{~s}$, one by one where the analogue correlated-double sampling circuits multiplex from one CCD to another. The N-FEE units digitise with high resolution 16-bit ADCs that provide low noise, high precision video processing in order to sample accurately. The N-FEE includes a power-supply filter PCB to reduce common-mode and differential-mode noise coupled into and out of the N-FEE. Low noise linear regulators are used to maximise immunity to incoming noise for the power supplies to the analogue circuitry; linear regulators are also used to derive the digital supplies. The NFEE has SRAM included to facilitate windowing masks to reduce the communication bandwidth and on-board storage required. Acquisition of house-keeping bias voltages and high resolution temperatures are implemented within the N-FEE for each CCD bias, CCD integrated temperature sensor and PCB temperature sensors. The N-FEE receives an external clock which is used to operate the FPGA within the electronics where jitter is controlled with the use of a phase locked loop to further maintain the signal to noise ratio and effective resolution.

Each CCD, when clocked by the FPGA employs a tri-level clocking scheme for the image clocks with the levels being $-\mathrm{ve}, 0 \mathrm{~V}$ and $+\mathrm{ve}$. Digital to analogue converters are used to set some of the CCD bias voltage levels which may be adjusted during the flight to compensate for radiation or other CCD ageing related effects. The nominal CCD operating temperature is 203 K. Post launch during the commissioning phase the temperature of the TOU will be varied between $\pm 10 \mathrm{~K}$ to identify the optimal point of focus for each camera. Thus the temperature of the CCDs from camera to camera will be in the range of $193 \mathrm{~K}$ to $213 \mathrm{~K}$. The typical readout for the normal cameras will utilise one lead-in row for electronic stabilisation, which is a row 
readout without performing a parallel transfer. Thirty rows and fifteen columns of overscan are included to enable inflight monitoring of CTI. The amount of overscan is based on measurements of the post irradiation performance performed by ESA [8].

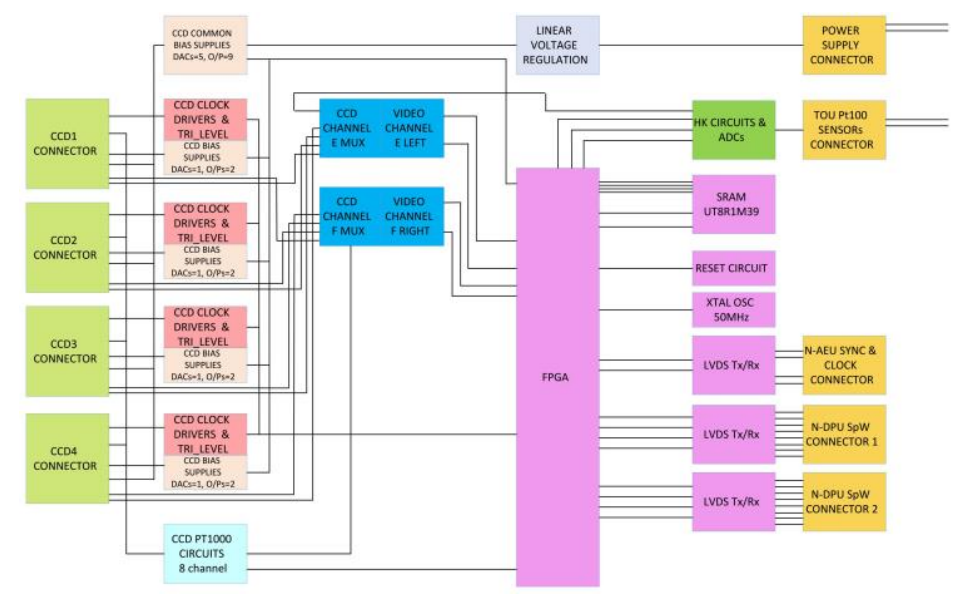

Figure 1: Block diagram of the EMv1 N-FEE

The volume of data produced by the twenty six cameras is too large to transmit in full, therefore pre-identified target stars are assigned an imagette, typical imagette size is $6 \times 6$ pixels for the normal cameras [1]. The majority of imagettes will be processed on board to provide a brightness measure. Only a limited number can be transferred to the ground. The Data Processing Unit (DPU) provides a look-up table to the N-FEE which dictates which pixels are transferred to the DPU which, in turn, performs basic photometric analysis. The resulting light curves, imagettes and centroid data are sent to the instrument control unit and are then transferred to the ground for further processing [1].

\subsection{Radiation Damage Implications}

The CCDs will suffer a decrease in photometric performance as a result of the space radiation environment [9], primarily through the formation of traps impacting charge transfer. This is as a result of both charge loss and by deferred charge tails which increase noise on a star due to charge tails from nearby stars in the same column and row. It is also important to know the amount of charge deposited by stars while the CCD is clocking, referred to as 'smearing', to allow it be to subtracted. The smearing is measured in the parallel overscan, and the measurement is obscured by deferred charge tails so these need to be removed. The exact impact of on PLATO measurements is currently under discussion, with information on some of the recent data generated to aid this discussion to be found in references 8 and 10. To ensure tools to correct for charge loss and charge tails being developed at LESIA, based on methods used to correct CTI in Euclid [11-12], are using appropriate trap parameters the CTI will be monitored in flight, with more detailed characterisations performed after solar flares.

A defect capable of trapping charge and increasing the CTI can be created when an energetic particle, typically a proton (solar or galactic in origin), displaces an atomic nucleus from the lattice. The resulting damage is dependent on particle type and energy, impurity levels within the CCD and the thermal history of the device post irradiation [13]. PLATOs launch will take place two years after the expected point of solar maximum during solar cycle 25 . Predictions of the intensity range from being comparable to cycle 24 [14] to being weaker [15]. If cycle 25 is similar to cycle 24 the environment experienced by PLATO will lead to a similar trend in the increase in CTI experience by Gaia [16], i.e. a steady increase populated by 
occasional sharp uplifts as a result of solar flares.

Typically the defects which impact charge transfer within an n-channel $\mathrm{CCD}$ are the phosphorous-vacancy (E-centre), oxygen-vacancy (A-centre), an unknown defect, and the divancancy composed of two adjacent vacancies [17-18]. The E-centre typically has the most impact on charge transfer in CCDs as it is the most abundant defect post irradiation. Emission time constants for the traps likely to impact charge transfer are shown in Figure 3, based on published values for trap energy levels and cross sections [17-18]. The impact a defect has on CTI is dependent on its emission time constant and the CCD timings. The timings are described by the time in which trapped charge can re-join the charge packet, $t_{r}$, dependent on the clock waveform used and a minimum and maximum time in which charge that is trapped could rejoin. The dwell time is the time the register clocks (serial) and image clocks (parallel) are static while the other is performing a charge transfer, the readout time is the time required to complete one readout and the integration time is the time set to collect incident charge.

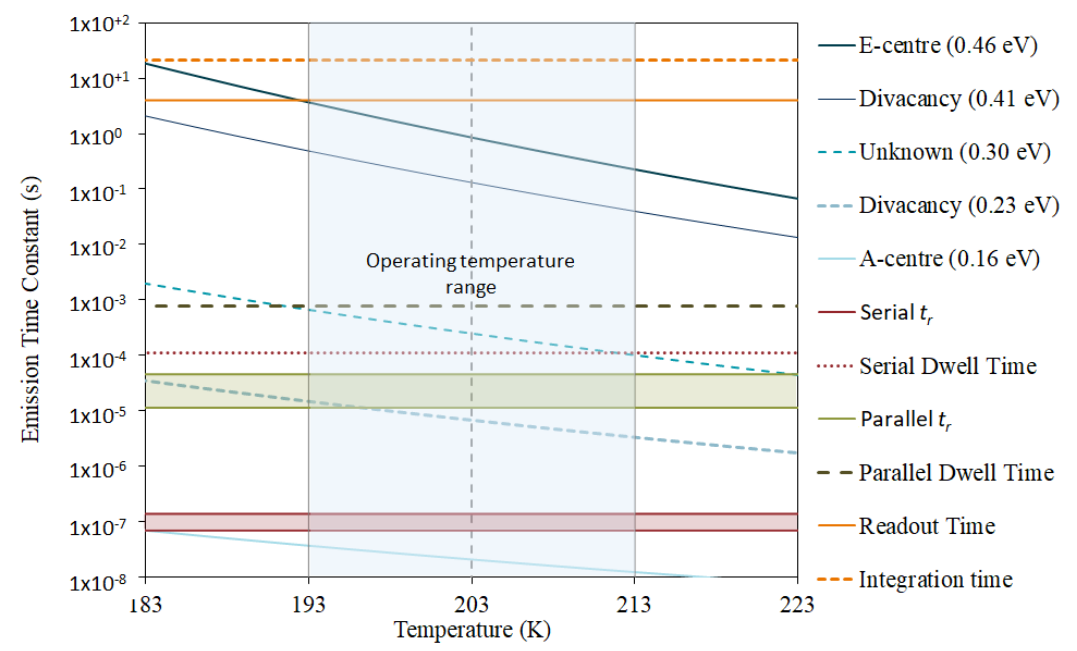

Figure 3: Defect emission time constants and the normal CCD operating timings as a function of temperature around the nominal PLATO CCD operating temperature of $203 \mathrm{~K}$

The impact of the E-centre on parallel transfer will increase with increasing temperature as these traps will start to capture charge multiple times during readout, the same is true for the divacancy at $0.41 \mathrm{eV}$ with less impact on charge transfer due to a lower number of traps being formed. This will lead to an increase in CTI at warmer temperatures. Prior to the start of the readout the majority of traps in the device will have released, in particular traps under the barrier phases, i.e. the electrodes not biased during the integration time, will likely be empty. The sky background will help suppress traps in the collecting phases and during charge transfer and the implementation of charge injection to further suppress the slow traps is currently under consideration. Sky background and the use of charge injection will also mitigate the impact of the unknown defect which will empty during the parallel dwell time (serial readout).

The parallel $t_{r}$ shown in Figure 3 indicates that the influence of the divacancy at $0.23 \mathrm{eV}$ will decrease as the influence from the unknown defect increases at higher temperatures, leading to a reduction in parallel CTI at warmer temperatures. To reduce the impact of the divacancy at $0.23 \mathrm{eV}$ on charge transfer, in particular at lower temperatures, tri-level clocking has been implemented in the latest version on the N-FEE. Tri-level clocking increases the probability that charge from fast traps can re-join the original charge packet [19]. As the temperature increases the impact of the A-centre on serial $t_{r}$ will reduce, giving slightly lower serial CTI at warmer 
temperatures. Due to the different camera operating temperatures the impact of a particular trap will be different from camera to camera, making it essential that each camera is treated individually for charge transfer correction.

\section{On Ground Characterisation}

The next stage of work is the investigation into the performance of the N-FEE described in section 1.2. This will include measurements of readout noise, bias, gain, gain thermal sensitivity and linearity saturation (the point at which the N-FEE saturates is higher than the CCDs FWC). These tests, with the exclusion of linearity saturation, will then be repeated using a CCD270 in a small test chamber with the N-FEE at room temperature. These tests will include the measurement of dark current, charge injection uniformity, cosmetic defects, CTI, FWC, charge to voltage conversion (CVF), and linearity. A second test system will then be used to perform more detailed optical investigations into device performance including the measurement of relative Quantum Efficiency (QE), Pixel Response Non-Uniformity (PRNU) and Intra-PRNU (IPRNU). Note that absolute QE measurements at a range of temperatures will be made by Teledyne e $2 \mathrm{v}$ on each CCD prior to delivery. The second test system has the option to place the $\mathrm{N}$-FEE inside the vacuum chamber which allows its performance at different temperatures to be assessed while holding the CCD at a constant temperature. These test systems will be used during the final stages of N-FEE development and for the initial characterisation of Engineering Model (EM) and Qualification Model (QM) versions of the N-FEE and CCD. Data will be collected at $\pm 10 \mathrm{~K}$ of the CCD nominal operating temperature in steps of $2.5 \mathrm{~K}$.

The process of characterisation, defined in PLATO as the generation of data which will be used in the future (e.g. for correction), will be performed with the one EM, one QM and with each of the Flight Models (FM). The characterisation of the N-FEE, without a CCD, will include the N-FEE tests described previously and will be performed in the optical test chamber. The N-FEE will then be connected to the equivalent model of CCD which will be mounted on the FPA, as shown in Figure 4, and placed inside the MSSL Optical Calibration facility, a $2.0 \mathrm{~m}$ diameter by $2.1 \mathrm{~m}$ long chamber developed to perform optical calibration of larger instruments. A complete electro-optical characterisation will then be performed for each camera. With the exception of IPRNU which will, due to the complexity and time required for the tests, only be performed on one FM CCD in the smaller optical test chamber. IPRNU is most likely to be similar for all devices since it is largely design specific.

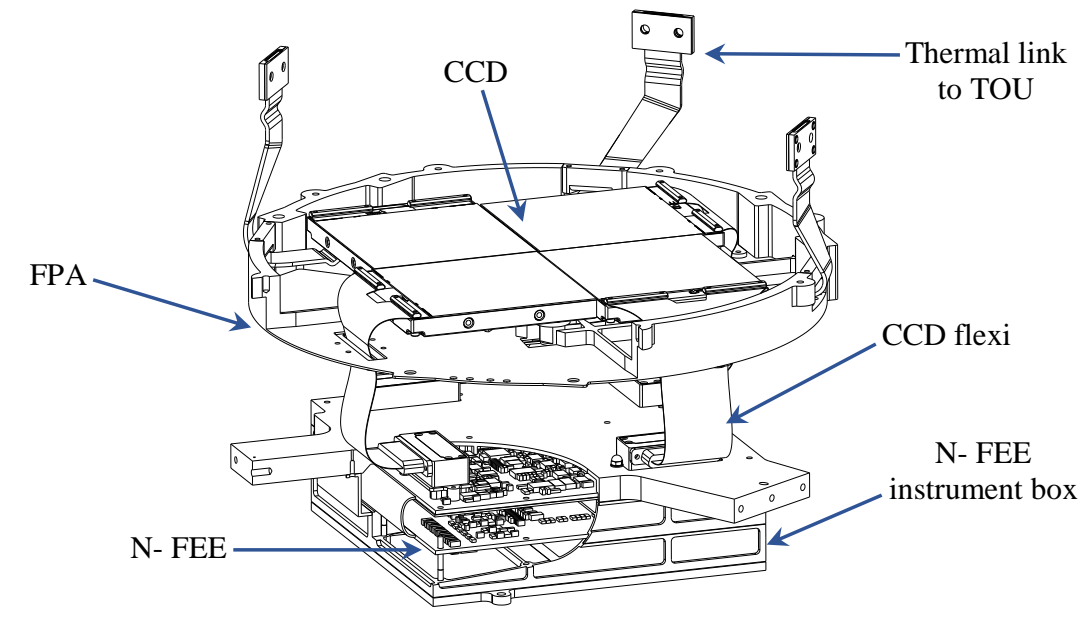

Figure 4: Mechanical drawing of the N-FEE, instrument box, FPA and CCDs 


\section{Inflight Test Strategy}

It is important for inflight characterisation to have minimal impact on the generation of science data. The monitoring and characterisation of $\mathrm{CCD}$ performance will take place on a ' $\mathrm{C}$ 'ontinuous activity based on the on-going science measurement and as part of ' $\mathrm{O}$ 'ccasional characterisation sequences. Occasional characterisation sequences will also be performed during the initial calibration phase, following large solar flares etc., and may be performed during manoeuvres to minimise impact to the science operations. In some cases of the occasional calibrations, 'N'ormal CCD clocking will be used to acquire the data. In other cases, 'S'pecial clocking modes will be required, and the N-FEE field-programmable gate array will be preprogrammed to implement these. In most cases extra data will be required to be processed and/or telemetered to obtain the measurement. The limitations placed on what can be achieved in orbit are that there is no on-board calibration lamp, there is no shutter and the N-CCDs are full-frame devices (the store and image section clock supplies are common in the N-FEE). It is planned to include a measurement of readout noise, dark current and sky background, cosmetics, FWC, linearity, CVF, PRNU, relative QE, an assessment of CCD Point Spread Function (PSF) and CTI for inflight monitoring. Absolute camera QE measures may be determined from the monitoring of 'standard' stars over a range of spectral classes.

A continual health check on the CCDs will be provided by monitoring the readout noise $(\mathrm{C}, \mathrm{N})$ using imagettes placed over the pre-scan pixels, thereby allowing any issues with a particular CCD to be quickly identified. The separation of dark current and sky background is complicated by the lack of a shutter, both can be monitored by placing imagettes in empty areas of sky $(\mathrm{C}, \mathrm{N})$ and by monitoring the amount of charge within the parallel overscan $(\mathrm{C}, \mathrm{N})$. An estimation of dark current could be found by accounting for TOU throughput and observations made in the same area of sky, this would require images taken with a longer integration times $(\mathrm{O}, \mathrm{N})$ and may only be possible towards the later stages of the mission when dark current generation will be higher. Dark current in the F-CCDs can be monitored using the store regions, and this could be used to aid in the estimation of dark current in the N-CCDs. The formation of defects can be monitored using normal science data $(\mathrm{C}, \mathrm{N})$ with the need to perform occasional full frame images at longer integration times $(\mathrm{O}, \mathrm{N})$.

The linearity can be monitored using data collected with different integration times $(\mathrm{O}, \mathrm{N})$ or by using a special clocking mode where no integration time is applied but an additional pause is introduced during each line transfer $(\mathrm{O}, \mathrm{S})$ to create a photon transfer curve. This will also provide a measurement of FWC and allow the CVF to be determined. The FWC and blooming can also be monitored using bright stars in normal operation $(\mathrm{C}, \mathrm{N})$ with occasional images with longer integration times $(\mathrm{O}, \mathrm{N})$. Long-term monitoring of star brightness can also be used as a monitor of CCD/FEE gain (C,N), but is affected by TOU transmission, CCD QE and CCD surface contamination. The PRNU can be measured using imagettes placed over empty areas of sky $(\mathrm{C}, \mathrm{N})$, however more accurate measurements would require longer integrations $(\mathrm{O}, \mathrm{N})$. It should be possible to separate sky background effects from PRNU effects by comparing images of the same area of sky from different CCDs. The relative QE can also be monitored in normal science data by looking at the evolution of signal (accounting for TOU throughput, CVF and FEE gain) (N,C). If sky background is used the accuracy of this measurement would be low, and so would require images to be taken with increased integration times $(\mathrm{O}, \mathrm{N})$. However, it is not expected that the sensitivity of individual pixels will change significantly during the mission except due to contamination effects, therefore monitoring of star brightness across the field (an essential part of normal operations) will provide larger scale sensitivity variations. 
Star image PSF is larger than CCD PSF, so it is not practical to use digitised star PSFs to investigate CCD PSF. CCD PSF could be monitored using incident low energy charged particles, however this is not practical to implement because of the complexity of event recognition in the on-board software compared with the benefit of the data. Therefore to investigate CCD PSF would require full field images to be transmitted to allow useful events to be identified on the ground $(\mathrm{O}, \mathrm{N})$.

\subsection{CTI Monitoring}

Currently there are a number of options under consideration to monitor the CTI and generate the information required for charge loss and charge tail correction. CTI measurements can be made using charged particle interaction, however as already described this would require event recognition software, and bright defects, which can give localised information, can only be monitored once they are created. Therefore the sources of charge under consideration to allow CTI to be measured are from stars, sky background and charge injection. Two options for using stars are under consideration. The first is to monitor the deferred charge tails using additional imagettes placed adjacent to a number of stars $(\mathrm{C}, \mathrm{N})$, as shown in Figure 5(a), some of which would be photometric standards. The tail from stars selected on the edge of the device would have imagettes placed in regions of overscan. The second option would be to sum the amount of charge deposited within the detector for individual stars using data generated during normal science operation $(\mathrm{C}, \mathrm{N})$, the same data being used to generate the light curves, and use the same technique employed during X-ray CTI measurements [20] to produce a scatter plot from which a value for CTI can be determined. The signal level of individual stars could be binned to provide a CTI value at different signal levels, with the initial images being used to set the baseline level i.e. where the CTI is equivalent to on ground performance.

(a)

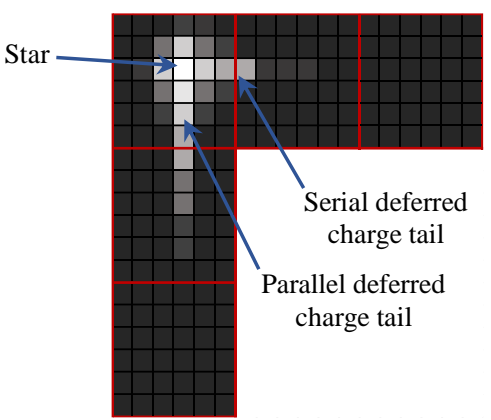

(b)

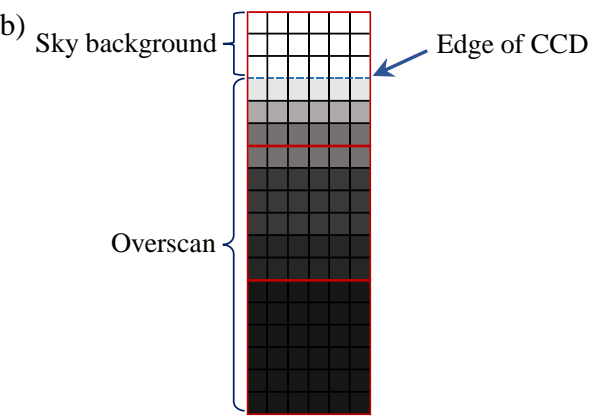

Figure 5: Imagette arrangement which could be used to analyse deferred charge tails from stars (a) and the deferred charge tails from the sky background in the overscan (b)

The sky background can be used to measure deferred charge using imagettes placed on the edge of the device in areas where there are no stars $(\mathrm{C}, \mathrm{N})$, Figure 5(b) for parallel and rotated by 90 degrees for serial. This provides a good measurement of the amount of deferred charge at a particular signal level using almost the same technique a typical on ground Extended Pixel Edge Response (EPER) measurement would use (i.e. a diffuse optical background) [20]. Imagettes could also be placed over the serial pre-scan pixels to monitor the amount of charge lost during the initial 25 transfers measured using the First Pixel Response (FPR) technique [20].

The use of charge injection increases the range of signals which can be used to create deferred charge tails within the overscan $(\mathrm{C}, \mathrm{S})$. Allowing trap parameters for both serial and parallel charge transfer (measured using EPER and FPR) to be determined, using imagettes placed as shown in Figure 6. The advantage of using charge injection as a monitor over the use 
of stars or the sky background is that it can provide a repeatable level of charge from which to make the CTI measurement. With the charge injection taking place in the overscan there is no reduction in image area and monitoring can take place with no impact on scientific operation. It will be possible to sweep the imagettes monitoring parallel deferred charge across the columns of the device in subsequent images allowing the charge loss across the entire device to be assessed. As with sky background imagettes could also be placed in the serial pre-scan to monitor the charge lost during the first 25 transfers.

This continual data collection will allow for the CTI to be monitored and provide part of the deferred charge tail to be used to update trap parameters for charge correction. However, more detailed characterisation will be performed periodically and after the identification of a step change in CTI after a solar proton event $(\mathrm{O}, \mathrm{S})$. This requires specific images for calibration, meaning an interruption to scientific data collection. The primary option is to take images with an increased amount of parallel and serial overscan.

(a)

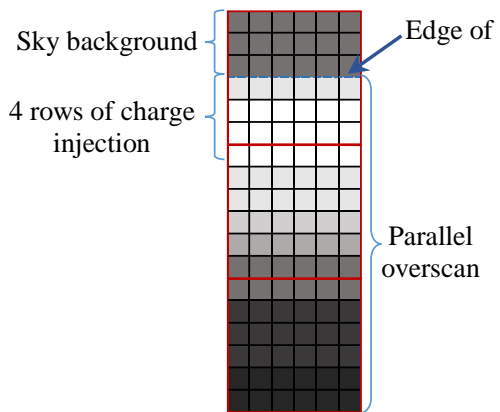

(b)

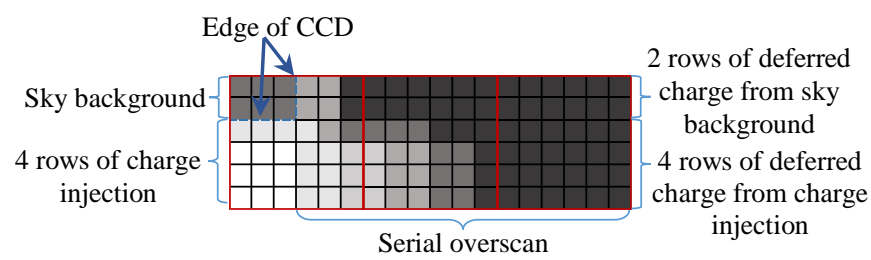

Figure 6: Imagette arrangement which could be used to analyse deferred charge tails and charge loss of charge injected into the parallel overscan, for parallel (a) and serial charge transfer (b)

The possibility of implementing variable gap injection [21] is also under consideration to enable charge loss and deferred charge to be investigated in one image $(\mathrm{O}, \mathrm{S})$. These images would be composed of 200 rows of injected charge followed by a gap without charge injection of between 100 and 4000 rows ( $78 \mathrm{~ms}$ to $\sim 3.1 \mathrm{~s})$ with the remaining CCD rows filled with charge injection. It is possible to increase the gap by performing additional transfers into the overscan or by the addition of a pause to the line transfer, it is only limited by the practicality and benefit. This method is particularly useful to probe traps with long emission times which can be difficult to investigate using deferred charge tails. The emission time constant of the E-centre at $193 \mathrm{~K}$ is around $3.7 \mathrm{~s}$, therefore this technique would only be able to investigate the E-centre (without extending the gap) down to around $199 \mathrm{~K}$. This relates to the point where the emission time constant is half of the maximum gap time.

\subsection{Inflight Defect Identification}

Trap pumping relies on moving charge backwards and forwards from one pixel to another for many thousand transfers, capture and release happen at specific points during this transfer related to the defect. Therefore depending on the defect type and where it is located it will either release charge back into the original charge packet (nothing identified), release it into the starting pixel or release it into the adjacent pixel charge is being clocked into creating a dipole. By varying the time at which pumping is performed, described by the phase time $t_{p h}$, the emission time constant of the trap can be determined [22]. The minimum $t_{p h}$ is set by the electrode capacitance, which determines the maximum speed of transfer due to the clock rise and fall times, and the maximum $t_{p h}$ is determined by the time available in which pumping can 
be performed. Data generated from trap pumping can be used to supplement information on traps from deferred charge and FPR measurements for charge correction, and this is the use of the data currently envisioned. The information could also be used to create a trap map of defects present in the CCD and a Monte Carlo based model used to perform correction, however the practicality of this would be limited by the inability to identify the location of slower traps and its value will require an assessment. A simple method of using trap pumping to correct for charge loss relies on moving the charge backwards and forwards using the same operational timings as normal readout, the assessment of this method however is still at an early stage [23].

If only defect monitoring is required then pumping within the serial register is optimal, allowing complete sweeps or repeats of a particular $t_{p h}$ value to be assessed within the same image. Currently a maximum cadence of $100 \mathrm{~s}$ has been set as the limit of interrupt. Therefore with one $t_{p h}$ value explored every two rows a $t_{p h}$ of between $1.0 \times 10^{-8}$ to $1.0 \times 10^{-4} \mathrm{~s}$ could be explored, which should be sufficient to probe the A-centre and divacancy at $0.23 \mathrm{eV}$.

\section{Conclusions}

The ground characterisation that will be performed in support of PLATO at MSSL will ensure that the best possible performance will be achieved for all of the cameras and generate the required information which can be used during science data processing. The monitoring of CCD performance inflight on a continuous basis will provide a health check and calibration update on the operation of the CCD without significantly impacting the collection of scientific data. With additional more detailed characterisation of CCD performance performed on an occasional basis, possibly during manoeuvres to minimise impact on science operation. The current options to monitor CTI and to ensure correction tools incorporate the latest trap parameters and quantities during flight have been described. The advantage of using charge injection, or sky background, is that the measurement techniques are the same as typical on ground characterisation allowing for a comparison to on ground data collected as part of the PLATO radiation damage characterisation.

The use of trap pumping, which has been used successfully during on ground testing [22] and in orbit [23] to investigate specific defects and their locations is also under consideration. The technique is an excellent source of information about defect formation and evolution and it would provide a good comparison between inflight observations and the on ground radiation damage testing. Trap pumping to monitor the formation of defects is also planned for implementation in the Euclid Visible Imager [24].

PLATO will generate a wealth of information identifying habitable worlds around bright stars that can then be investigated further using on ground observations, and perhaps one day be the target of interstellar exploration. However the detection of Earth-like planets requires PLATO to operate near its performance limit. The development of the FEE aims to maximise the performance of the CCD, ensuring that PLATO will generate excellent science throughout the mission and to provide the ability to characterise device performance during the mission. The FEE itself builds on the heritage at MSSL in the production of flight electronics, including Integral-OMC, optical monitors on XMM-Newton and SWIFT, Hinode-EIS, developments for Eddington, Gaia and Euclid. The FEE developed for PLATO will itself be adapted for use in the ESA Solar Wind Magnetosphere Ionosphere Link Explorer (SMILE) soft X-ray imager [25]. 


\section{Acknowledgments}

The authors would like to thank the PLATO CCD working group for their support during this study.

\section{References}

[1] H. Rauer, C. Catala, C. Aerts, et al., The PLATO 2.0 mission, 2014 Experimental Astronomy vol. 38 Issue 1-2, 249-330.

[2] R. Ragazzoni, D. Magrin, H. Rauer, et al., PLATO: a multiple telescope spacecraft for exo-planets hunting, 2016 Proc. SPIE 9904.

[3] J. Endicott, A. Walker, S. Bowring, et al., Charge-coupled devices for the ESA PLATO M-class Mission, 2012 Proc. SPIE 8453.

[4] G. W. Henry, G. W. Marcy, R. Paul Butler, and S. S. Vogt, A Transiting "51 PEG-LIKE" Planet, 2000 The Astrophysical Journal 529 L41-L44

[5] M. G. Hidas, M. C. B. Ashley, J. K. Webb et al., The University of New South Wales Extrasolar Planet Search: methods and first results from a field centred on NGC 6633, 2005 Mon. Not. R. Astro. Soc. vol. 360 Issue 2 703-717.

[6] S. Udry, C. Lovis, F. Bouchy, et al., Exoplanet Science with the European Extremely Large Telescope. The Case for Visible and Near-IR Spectroscopy at High Resolution, 2014 arXiv:1412.1048.

[7] M. J. S. Belton, K. P. Klaasen, M. C. Clary, et al., The Galileo Solid-State Imaging Experiment, 1992 Space Science Reviews vol. 60 p. 413-455

[8] T. Prod'homme, P. Verhoeve, T. Beaufort, et al., Technology validation of the PLATO CCD at ESA, 2016 Proc. SPIE 9915.

[9] E. G. Stassinopoulos and J. P. Raymond, The Space Radiation Environment for Electronics, 1988 Proc. of the IEEE, vol. 76, pp. 1423-42.

[10] P. Verhoeve, T. Prod'homme, T. Oosterbroek, et al., Optical characterization of the Plato CCD at ESA, 2016 Proc. SPIE. 9915.

[11] H. Israel, R. Massey, T. Prod'homme, et al., How well can Charge Transfer Inefficiency be corrected? A parameter sensitivity study for iterative correction, 2015 Mon. Not. R. Astron. Soc. vol. 453, pp. 561-580.

[12] A. Short, C. Crowley, J. de Bruijne, and T. Prod'homme, An analytical model of radiation-induced charge transfer inefficiency for CCD detectors, 2013 Mon. Not. R. Astron. Soc. Vol. 430, pp. 30783085 .

[13] J. R. Srour, C. J. Marshall, and P. W. Marshall, Review of Displacement Damage Effects in Silicon Devices, 2003 IEEE Trans. Nucl. Sci., vol. 50 no. 3.

[14] R. H. Cameron, J. Jiang and M. Schüssler, Solar Cycle 25: Another Modertae Cycle?, 2016 The Astrophysical Journal Letters, 823:L22.

[15] N. Gopalswamy, S. Yashiro and S. Akiyama, Unusual Polar Conditions in Solar Cycle 24 and their Implications for Cycle 25, 2016 The Astrophysical Journal Letters, 823:L15. 
[16] C. Crowley, A. Abreu, R. Kohley, et al., Radiation effects on the Gaia CCDs after 30 months at L2, 2016 Proc. SPIE 9915.

[17] P. Pichler, Intrinsic point defects, impurities, and their diffusion in silicon, Computational Microelectronics Springer Germany 2004.

[18] A. D. Holland, The effect of bulk traps in proton irradiated EEV CCDs, 1993 Nucl. Inst. Meth. A vol. 326 no. 1-2 pp. 335-343.

[19] N. J. Murray, D. J. Burt, A. D. Holland, et al., Multi-level parallel clocking of CCDs for: improving charge transfer efficiency, clearing persistence, clocked anti-blooming, and generating low-noise backgrounds for pumping, 2013 Proc. SPIE 8860.

[20] J. R. Janesick, Scientific Charge Coupled Devices, SPIE Press Washington 2001.

[21] G. R. Hopkinson, A. Short, C. Vetel, et al., Radiation Effects on Astrometric CCDs at Low Operating Temperatures, 2005 IEEE Trans. Nucl. Sci., vol. 52 no. 2.

[22] D. J. Hall, N. J. Murray, A. D. Holland, et al., Determination of In Situ Trap Properties in CCDs Using a "Single-Trap Pumping" Technique, 2014 IEEE Trans. Nucl. Sci., vol. 61 no. 4.

[23] J. P. D. Gow and N. J. Murray, Simplified Charge Transfer Inefficiency Correction in CCDs by Trap-Pumping, 2016 Proc. SPIE 9915.

[24] M. Cropper, S. Pottinger, S. Niemi, et al., VIS: the visible imager for Euclid, 2016 Proc. SPIE 9904.

[25] W. Raab, G. Branduardi-Raymont, C. Wang, et al., SMILE: a joint ESA/CAS mission to investigate the interaction between the solar wind and Earth's magnetosphere, 2016 Proc. SPIE 9905. 